\title{
Impurity-Mediated Early Condensation of a Charge Density Wave in an Atomic Wire Array
}

\author{
Han Woong Yeom, ${ }^{*}, * \neq$ Deok Mahn Oh, ${ }^{\dagger,}$ Stefan Wippermann, ${ }^{\S}$ and Wolf Gero Schmidt ${ }^{\perp}$ \\ ${ }^{\dagger}$ Center for Artificial Low Dimensional Electronic Systems, Institute for Basic Science (IBS), Pohang 790-784, Korea \\ ${ }^{\ddagger}$ Department of Physics, Pohang University of Science and Technology, Pohang 790-784, Korea \\ ${ }^{\S}$ Interface Chemistry and Surface Engineering Department, Max-Planck-Institute for Iron Research GmbH, Max-Planck-Straße 1, \\ 40237 Düsseldorf, Germany \\ ${ }^{\perp}$ Lehrstuhl für Theoretische Physik, Universität Paderborn, 33098 Paderborn, Germany
}

\section{Supporting Information}

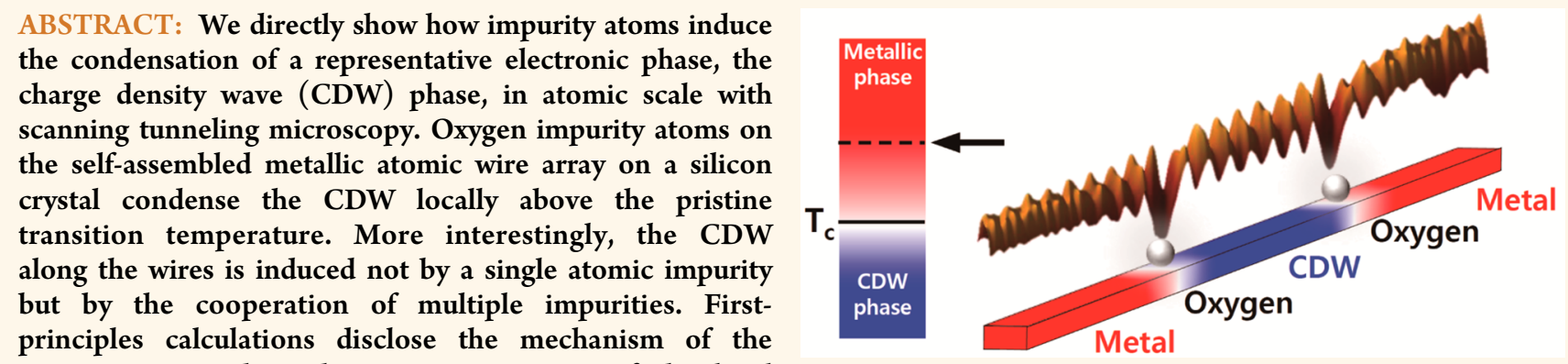
cooperation as the coherent superposition of the local lattice strain induced by impurities, stressing the coupled electronic and lattice degrees of freedom for the CDW. This opens the possibility of the strain engineering over electronic phases of atomic-scale systems.

KEYWORDS: atomic wire, impurity, charge density wave, phase transition, scanning tunneling microscopy

$\mathrm{E}$ ffects of impurities in phase transitions are important and widely studied, which are often drastically enhanced in nanoscale systems. An impurity is generally believed to prohibit long-range orders ${ }^{1}$ or induce the condensation of low-temperature phases below the transition temperature $\left(T_{\mathrm{c}}\right)^{2,3}$ However, the dominant majority of previous studies focused not on individual impurities but on statistical ensembles of them, and the direct atomic-scale observation of the impurity-mediated condensation of phases has rarely been reported. In electronic transitions of condensed matter systems, the impurity effect is also believed to be crucial. ${ }^{4-6}$ At low temperature the impurity and defect distributions were found correlated with the superconducting phase inhomogeneity 4,5 and with domain structures of charge density wave (CDW) phases. ${ }^{6}$ These phenomena would obviously be connected to the initial condensation of electronic phases with a finite density of atomic-scale impurities, which are usually ill characterized and whose microscopic interactions with electronic phases are elusive. ${ }^{4-6}$ Moreover, the impuritymediated condensation of these electronic phases has not been directly and clearly probed near $T_{c}$.

In this study, we made important progress in addressing the impurity-induced condensation of the electronic phase especially in a quasi-one-dimensional (quasi-1D) system. We access a quasi-1D CDW transition near $T_{c}$ with atomic resolution for a well-characterized and controllable atomic impurity coupled actively with the CDW. We could directly observe the atomic-scale condensation of the CDW phase above $T_{c}$ of the clean system as induced by impurities. Moreover, the microscopic mechanism of the early condensation was transparently clarified through first-principles calculations. The CDW system we choose is a quasi-1D metallic system formed on a semiconductor surface of $\mathrm{Si}(111){ }^{7}$ Indium atoms deposited at an elevated temperature self-organize into a perfectly ordered array of metallic atomic wires; two zigzag In chains form a unit wire separated by Si zigzag chains (Figures $1 \mathrm{~b}$ and $2 \mathrm{a}){ }^{8}$ These wires have three $1 \mathrm{D}$ metallic bands, which, together with a strong coupling to the lattice, drive a metalinsulator transition into a periodically distorted insulating state at $125 \mathrm{~K}^{7,9}$ In this CDW phase, the dimerization along In zigzag chains and the sheer displacement between two neighboring chains result in the formation of the characteristic hexagon structure (Figures $1 \mathrm{c}$ and $2 \mathrm{~b}$ ). ${ }^{10}$

This CDW system confined within a single atomic layer on the surface has no interlayer coupling, in contrast to conventional bulk CDW materials. This makes the direct

Received: September 18, 2015

Accepted: December 4, 2015

Published: December 4, 2015 
(a)

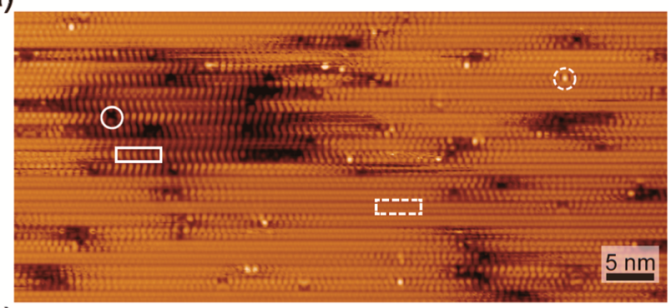

(b)

(c)
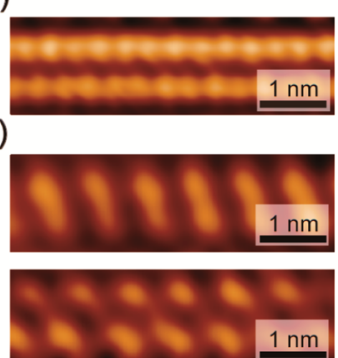

(d)

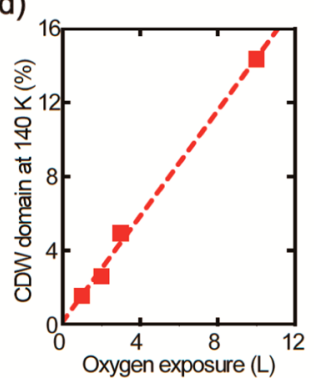

Figure 1. CDW of indium atomic wires with oxygen impurities. (a) STM topographic image after $3 \mathrm{~L}$ oxygen dose onto the In atomic wires at $140 \mathrm{~K}\left(>T_{\mathrm{c}}=125 \mathrm{~K}\right)$. The image shows the bright metallic parts [dashed box, enlarged in (b)] and dark local CDW condensates [box, enlarged in (c)] coexisting. The impurities are bright and dark spots (solid and dashed circles, respectively). Imaging conditions are $V_{\text {bias }}=-0.5 \mathrm{~V}[+0.5 \mathrm{~V}$ for the empty state image at the bottom of $(\mathrm{c})]$ and $I=100 \mathrm{pA}$. (d) Total area of the CDW domains as a function of the oxygen exposure.

(a)

(c)
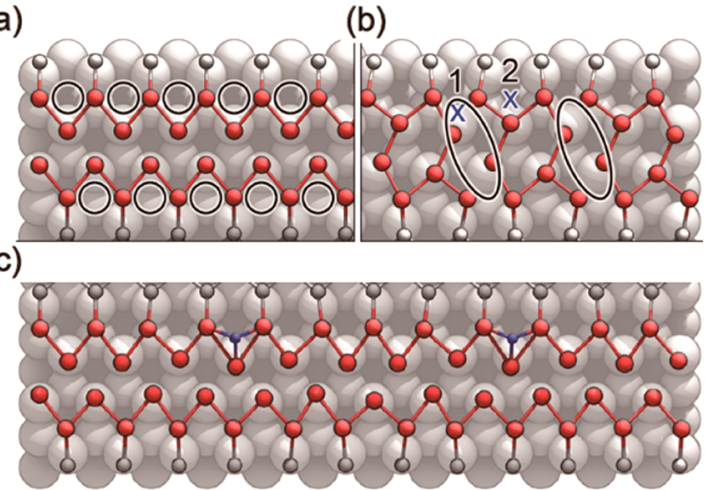

(d)

(e)

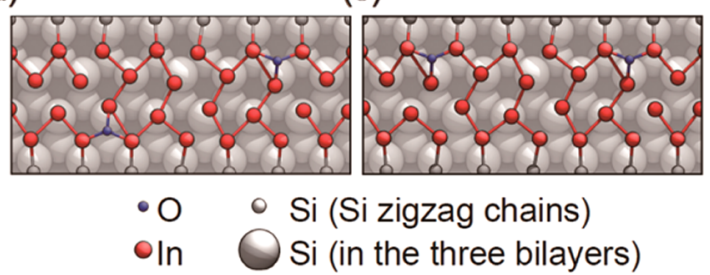

Figure 2. Atomic structures of In atomic wires with oxygen impurities. The atomic structures of (a) the metallic In wire above $T_{c}$ and (b) the wire in the CDW state below $T_{c}$. The circles (ovals) represent the protrusions in filled state STM images shown in Figure $1 b$ (Figure 1c). $X$ marked 1 and 2 in (b) are two different adsorption sites of the $\alpha$ impurity structure on the hexagonal structure, where on site 1 (2) the hexagonal structure is kept (inverted to the chain structure). (c) Calculated structure of an In atomic wire with two $\alpha$ impurities an odd multiple of $a_{0}$ apart $\left(5 a_{0}\right)$ which exhibits only the minor local distortion. The calculated structures with two impurities are even a multiple of $a_{0}$ apart $\left[4 a_{0}\right.$, (d) and (e)], which yield the hexagon CDW structure between impurities. observation of the impurity effect substantially easier; the impurity can easily be introduced and exposed as adsorbates, which can be characterized fully and controlled systematically, in stark contrast to impurities in bulk systems. ${ }^{11,12}$ Indeed, various impurity atoms were investigated previously such as $\mathrm{H}$, In, $\mathrm{Pb}, \mathrm{Na}$, and $\mathrm{Co}^{13-17}$ They commonly suppressed the local CDW order, ${ }^{15-17}$ causing the inhomogeneous phase separation and lowering $T_{c}$. This is consistent with the general wisdom of impurity effects ${ }^{1-3}$ as established in various electronic crystals. ${ }^{4,5}$ However, they do not provide any new insight into the CDW condensation. In contrast, the $\mathrm{CDW} T_{\mathrm{c}}$ was recently reported to increase proportional to the oxygen dose $^{16,17}$ The pristine In wires were found to be close to the optimal doping, and the electron or hole doping by oxygen adsorbates was found to be marginal. ${ }^{18}$ This suggests the existence of a novel local impurity-CDW interaction beyond the doping effect. ${ }^{19}$

\section{RESULTS AND DISCUSSION}

Figure 1a shows the In wires at $140 \mathrm{~K}$, above the $T_{\mathrm{c}}$ of $125 \mathrm{~K}$, with a 3 langmuir dose of $\mathrm{O}_{2}$. The corrugationless parts of the wires with the bright contrast are in the undistorted metallic phase, as enlarged in Figure $1 b .^{20}$ The short stripes and wider patches in the dark contrast are within the CDW state, ${ }^{20}$ as confirmed by the distinct STM images (Figure 1c) of In hexagons. The CDW gap on these stripes and patches are confirmed as discussed below. Namely, CDW already condenses locally at $140 \mathrm{~K}$ above $T_{c}$. There is a linear relationship between the total area of the CDW condensates and the oxygen dose (Figure 1d). This apparently indicates that the local CDW state is induced by oxygen impurities. The local CDW condensates exhibit only marginal fluctuations, suggesting further that they are pinned by impurities.

The oxygen impurities are found as isolated dark or bright spots, as shown in Figures 1 and 3a,b. The atomic structures of oxygen impurities were established in detail in previous works (see Supporting Information Figure S1). ${ }^{21,22}$ The dark impurity is the dominant species, called $\alpha$, where the oxygen atom adsorbs on one In zigzag chain to bond with three neighboring In atoms (Figures $2 \mathrm{c}$ and $3 \mathrm{a}$ ). The minority species appearing as a bright spot results from an activated process of the oxygen incorporation below the In layer (Figure $3 \mathrm{~b}$ and Supporting Information Figure S1). ${ }^{21}$ To our surprise, these isolated impurities and the isolated vacancy defects (see Supporting Information Figure S2) do not induce CDW condensates, but the $\times 2$ lattice distortion appears with a decaying amplitude from them. ${ }^{21}$ These distortions are distinct from the CDW hexagon in topography (Figure $3 \mathrm{~d}-\mathrm{f}$ ) and not insulating at all in spectroscopy (right panel of Figure $3 \mathrm{~h}$ ). Note that there can in principle be various different $\times 2$ lattice distortions since the present In wire system has four In atomic chains within a single wire. In stark contrast, in between two adjacent $\alpha$ impurities, the $\mathrm{CDW}$ hexagons are formed (Figure $3 \mathrm{~d}-\mathrm{f}$ ) with a clear energy gap (left panel of Figure $3 \mathrm{~h}$ ). We checked every isolated CDW stripe and found that the $\alpha$ impurity and another type of defect of the pristine surface (see Figure $4 \mathrm{c}$ and Supporting Information Figure S2) ${ }^{23}$ couple with the CDW. When two of them are within a distance of about 20 unit cells or shorter, they form CDW stripes (see Supporting Information Figure S3).

Beyond single wires, small CDW patches can be formed as seeded by a CDW stripe and other $\alpha$ impurities in a neighboring wire as shown in Figure 4a. This can be extended to wider CDW patches across a few wires with several $\alpha$ 
(a)

(b)
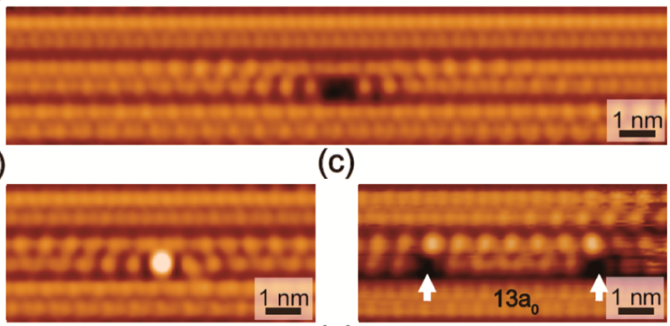

(d)

(c)

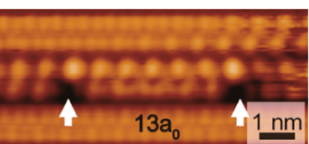

(e)

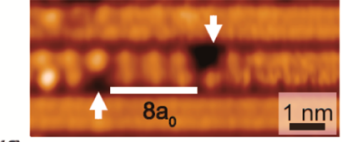

(f)

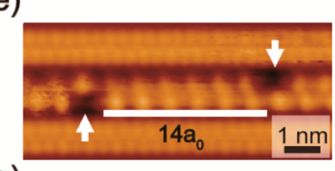

(g)

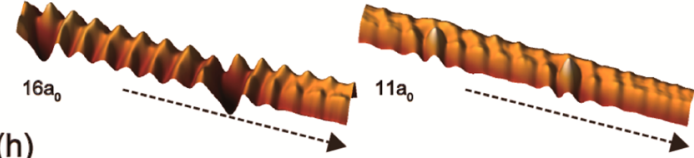

(h)

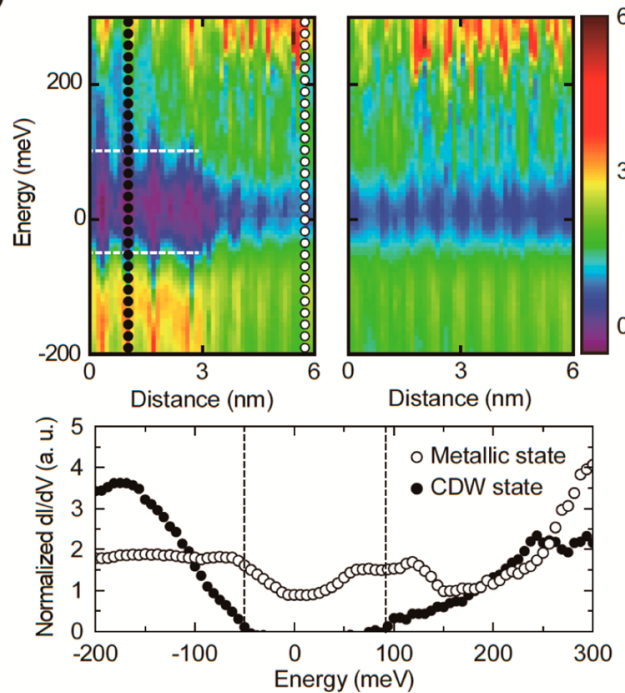

Figure 3. Single and double oxygen impurities and the formation of the CDW condensates. ( $a$ and $b$ ) STM images of oxygen impurities in two different adsorption structures, $\alpha$ and $\beta$, respectively, with lattice distortions around them. (c-f) STM images of two adjacent oxygen impurities (indicated by arrows, $\alpha$ structure) without (c) and with [(d), (e), and (f)] CDW hexagons (underlined in $\mathrm{d}$ and $\mathrm{e}$ ) formed in between. The distances between the impurities are $13 a_{0}$, $8 a_{0}, 14 a_{0}$, and $16 a_{0}$, respectively. (g) In atomic wire between two $\beta$ impurities, which do not induce a CDW (distance between two $\beta$ impurities is $\left.11 a_{0}\right)$. Imaging conditions are $V_{\text {bias }}=-0.5 \mathrm{~V}$ and $I=$ $100 \mathrm{pA}$. (h) Spatially and energetically resolved normalized differential conductance $(\mathrm{d} I / \mathrm{d} V)$ taken along the dashed arrows in (f) and $(\mathrm{g})$. Two representative $\mathrm{d} I / \mathrm{d} V$ curves for the insulating CDW and metallic parts are shown in solid and open circles. The CDW energy gap (dashed lines) exists only for the CDW hexagons in between two $\alpha$ impurities.

impurities clustered (Figure 4b). For a larger patch, a CDW stripe (boxed in Figure 4c) can be induced on a wire without an impurity when sandwiched between two CDW stripes bounded by impurities. These few cases can explain most of the impurity-CDW configurations (see Supporting Information Figure S4). That is, there exist only two apparent interactions to condense a CDW, the cooperation of two neighboring impurities within a single wire and the interwire coupling with neighboring CDW stripes. While the interwire interaction of (a)

(b)

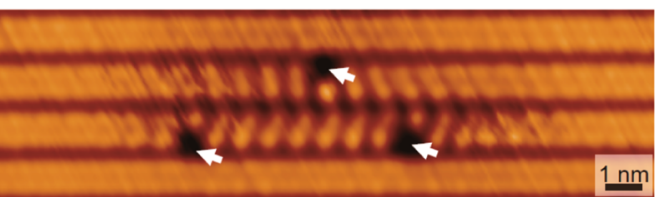

(c)
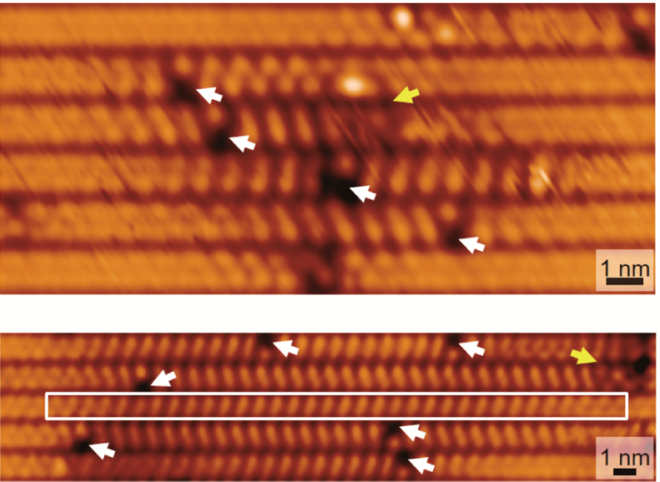

Figure 4. CDW patches formed by impurity clusters. (a) Small CDW patch composed two CDW stripes around three $\alpha$ impurities. (b) Larger patch with several $\alpha$ impurities clustered and a single vacancy defect (yellow arrow). (c) Much larger patch, which contains a CDW stripe (box) without any terminating impurity but sandwiched by neighboring CDW stripes. Imaging conditions are $V_{\text {bias }}=-0.5 \mathrm{~V}$ and $I=100 \mathrm{pA}$.

the CDW in the quasi-1D systems is obviously expected and apparent in the $2 \mathrm{D}$ ordering below $T_{\mathcal{c}}{ }^{6}$ the microscopic mechanism underlying the cooperation of two impurities remains to be clarified.

In order to clarify this microscopic mechanism, we performed first-principles calculations with single (Figure 2a and b) and double (Figure 2c-e) impurities. As reported previously, isolated oxygen impurities induce the local $\times 2$ lattice distortions but not the CDW hexagons. ${ }^{21}$ These local lattice distortions do not open a band gap as observed in the experiment (see Figure $3 \mathrm{~g}$ for example). In clear contrast, two adjacent impurities can induce CDW hexagons depending on their distance. When two impurities have a distance of an even multiple of $a_{0}$ (Figure $2 \mathrm{~d}, a_{0}$ is a Si lattice constant, $0.384 \mathrm{~nm}$ ), being commensurate with the CDW periodicity, hexagons are readily formed, but not in the other case of an odd multiple of $a_{0}$ (Figure 2c), being incommensurate with a CDW. In the former case, the lattice distortions of the neighboring oxygen impurities coherently overlap to make the hexagon formation energy lower. This is due to the fact that the CDW hexagon is based on the dimerization of In atoms of the outer In chains (In atoms bonded with $\mathrm{Si}$ zigzag chains), 9,10 and the oxygen adsorbate helps this dimerization by pulling two neighboring In atoms closer. The formation energy of hexagons is lowered by two cooperating oxygen atoms that are an even multiple of $a_{0}$ apart (8 and $30 \mathrm{meV}$, respectively, for the configurations in Figure $2 \mathrm{~d}$ and e). In contrast, In hexagons are not even metastable when bracketed between $\mathrm{O}$ atoms that are an odd multiple of $a_{0}$ apart; the lattice distortions by two impurities (Figure 2c) lead to only small modulations, in contrast to the hexagon structure. This is clearly observed in the experiment with the very clear distinction between the impurity pairs of odd and even multiples of $a_{0}$ apart (Figure $3 c$ and $f$, respectively). The coupling mechanism of the oxygen impurity with the CDW is simply based on the proper bond length 
modification due to the impurity and the coherent superposition of the strain field imposed by multiple impurities. In the present case, a strain effect is very natural, since the CDW is based on the electron-lattice interaction.

\section{CONCLUSION}

To the best of our knowledge, there had been no previous work to address directly the atomic-scale condensation process of electronic crystals such as CDWs and superconductivity ${ }^{4,5}$ by impurities. Most of the previous works for CDWs investigated the relationship of impurities and defects with the frozen CDW domains well below transition temperatures. Recently, the defect-induced CDW condensation was observed for a $2 \mathrm{D}$ $\mathrm{CDW}$ material of $2 \mathrm{H}-\mathrm{NbSe}_{2} .{ }^{24}$ However, in this case, the local electronic transition was not identified and the microscopic mechanism of the condensation is not disclosed. A similar study was reported for the phase transition on a $\mathrm{Sn} / \mathrm{Ge}(111)$ surface, ${ }^{25}$ which was, however, shown to be a simple structural order-disorder transition without a metal-insulator transition in later studies. ${ }^{26}$ While the impurity interaction with the conventional superconductors, suppressing the superconductivity for magnetic impurities, is well understood, ${ }^{27,28}$ the case for high-temperature superconductivity ${ }^{27,28}$ is elusive. ${ }^{5}$ In these materials, the superconductivity is even entangled with the $\mathrm{CDW}$ order, which in turn is closely coupled to impurities. ${ }^{29}$ Thus, further studies are required to understand the interplay of impurities, the CDW, and unconventional superconductivity. Therefore, the present case of the enhanced electronic order by atomic-scale impurities and the transparent microscopic mechanism is unique. The importance of the local strain field, as revealed here, was recently recognized also for twodimensional electronic systems of CDW of $2 \mathrm{H}-\mathrm{NbSe}_{2}{ }^{30}$ graphene, $^{31}$ and surface states of topological insulators. ${ }^{32}$ In those cases, however, the sources of local strains are ill defined and could not be controlled. In contrast, the present work with adsorbates provides controllability and, thus, takes steps forward to the atomic-scale strain engineering of important electronic phases.

\section{METHODS}

Sample Preparation. The sample was prepared in ultra-highvacuum conditions (UHV, the base pressure is $1.0 \times 10^{-10}$ Torr). The clean $\mathrm{Si}(111) 7 \times 7$ substrate was prepared by cycles of annealing and flashing up to $1500 \mathrm{~K}$ of the n-doped $\mathrm{Si}(111)$ wafer. The array of In atomic wires was prepared by depositing one monolayer of In onto a $\mathrm{Si}(111) 7 \times 7$ surface kept at $570 \mathrm{~K}^{7}$ Oxygen molecules were dosed onto the In atomic wire array at room temperature by backfilling the chamber through a precision leak valve. The $\mathrm{O}_{2}$ dose is expressed in langmuirs $\left(1 \mathrm{~L}=1.0 \times 10^{-6}\right.$ Torr $\left.\mathrm{s}\right)$.

STM and STS Measurements. STM and STS measurements were performed using a commercial cryogenic UHV STM system (Unisoku) at $140 \mathrm{~K}$ and a base pressure $5.0 \times 10^{-11}$ Torr. The electrochemically etched tungsten tips were cleaned by e-beam heating in the UHV chamber. The STM topography was measured in a constant-current mode, and the STS and the $\mathrm{d} I / \mathrm{d} V$ signals were obtained by a standard lock-in technique with a modulation of frequency $f=1 \mathrm{kHz}$ and amplitude of $30 \mathrm{mV}_{\text {rms }}$.

DFT Calculations. Density functional theory calculations were performed using the local density approximation (LDA) as implemented in the Vienna ab Initio Simulation Package (VASP). In the LDA calculations, indium $4 \mathrm{~d}$ electrons were treated as core electrons. The surface was modeled using three Si bilayers within a 12 $\times 8$ lateral unit cell. The Brillouin zone integration was obtained using a $k$-point sampling equivalent to 256 points within a $1 \times 1$ unit cell.

\section{ASSOCIATED CONTENT}

\section{Supporting Information}

The Supporting Information is available free of charge on the ACS Publications website at DOI: 10.1021/acsnano.5b05925.

Structure models, STM images, and calculated STM images of oxygen impurities on the In atomic wire; a large STM topographic image of the oxygen-dosed In atomic wire array on the $\mathrm{Si}(111)$ surface at $140 \mathrm{~K}$; STM topographic images of isolated CDW segments between two $\alpha$ oxygen impurities with different lengths; STM topographic images of several CDW patches (PDF)

\section{AUTHOR INFORMATION}

\section{Corresponding Author}

*E-mail: yeom@postech.ac.kr.

\section{Notes}

The authors declare no competing financial interest.

\section{ACKNOWLEDGMENTS}

This work was supported by Institute for Basic Science (IBSR014-D1). The numerical calculations were performed using grants of computer time from the Paderborn Center for Parallel Computing $\left(\mathrm{PC}^{2}\right)$ and the Hochstleistungs-Rechenzentrum Stuttgart (HLRS). The Deutsche Forschungsgemeinschaft (DFG) is acknowledged for financial support (FOR1700). H.W.Y. established the basic concept, designed the experiments, and wrote the manuscript with other authors. D.M.O. performed the STM experiments. S.W. performed the theoretical calculations with the help of W.G.S.

\section{REFERENCES}

(1) Imry, Y.; Ma, S.-k. Random-Field Instability of the Ordered State of Continuous Symmetry. Phys. Rev. Lett. 1975, 35, 1399-1401.

(2) Imry, Y.; Wortis, M. Influence of Quenched Impurities on FirstOrder Phase Transitions. Phys. Rev. B: Condens. Matter Mater. Phys. 1979, 19, 3580-3585.

(3) Aizenman, M.; Wehr, J. Rounding of First-Order Phase Transitions in Systems with Quenched Disorder. Phys. Rev. Lett. 1989, 62, 2503-2506.

(4) Balatsky, A. V.; Vekhter, I.; Zhu, J.-X. Impurity-Induced States in Conventional and Unconventional Superconductors. Rev. Mod. Phys. 2006, 78, 373-433.

(5) Alloul, H.; Bobroff, J.; Gabay, M.; Hirschfeld, P. J. Defects in Correlated Metals and Superconductors. Rev. Mod. Phys. 2009, 81, $45-108$.

(6) Grüner, G. Density Waves in Solids; Addison-Wesley Publishing Company, Advanced Book Program, 1994.

(7) Yeom, H. W.; Takeda, S.; Rotenberg, E.; Matsuda, I.; Horikoshi, K.; Schaefer, J.; Lee, C. M.; Kevan, S. D.; Ohta, T.; Nagao, T.; et al. Instability and Charge Density Wave of Metallic Quantum Chains on a Silicon Surface. Phys. Rev. Lett. 1999, 82, 4898-4901.

(8) Bunk, O.; Falkenberg, G.; Zeysing, J. H.; Lottermoser, L.; Johnson, R. L.; Nielsen, M.; Berg-Rasmussen, F.; Baker, J.; Feidenhans'l, R. Structure Determination of the Indium-Induced Si $(111)-(4 \times 1)$ Reconstruction by Surface X-Ray Diffraction. Phys. Rev. B: Condens. Matter Mater. Phys. 1999, 59, 12228-12231.

(9) Ahn, J. R.; Byun, J. H.; Koh, H.; Rotenberg, E.; Kevan, S. D.; Yeom, H. W. Mechanism of Gap Opening in a Triple-Band Peierls System: In Atomic Wires on Si. Phys. Rev. Lett. 2004, 93, 106401.

(10) González, C.; Ortega, J.; Flores, F. Metal-Insulator Transition in One-Dimensional In Chains on $\mathrm{Si}(111)$ : Combination of a Soft Shear Distortion and a Double-Band Peierls Instability. New J. Phys. 2005, 7, 100.

(11) Wu, X. L.; Lieber, C. M. Direct Characterization of ChargeDensity-Wave Defects in Titanium-Doped $\mathrm{TaSe}_{2}$ by Scanning 
Tunneling Microscopy. Phys. Rev. B: Condens. Matter Mater. Phys. 1990, 41, 1239-1242.

(12) Dai, H.; Lieber, C. M. Solid-Hexatic-Liquid Phases in TwoDimensional Charge-Density Waves. Phys. Rev. Lett. 1992, 69, 15761579.

(13) Hupalo, M.; Chan, T.-L.; Wang, C. Z.; Ho, K.-M.; Tringides, M. C. Interplay between Indirect Interaction and Charge-Density Wave in Pb-Adsorbed In $(4 \times 1)$-Si(111). Phys. Rev. B: Condens. Matter Mater. Phys. 2007, 76, 045415.

(14) Liu, C.; Uchihashi, T.; Nakayama, T. Self-Alignment of Co Adatoms on In Atomic Wires by Quasi-One-Dimensional ElectronGas-Meditated Interactions. Phys. Rev. Lett. 2008, 101, 146104.

(15) Morikawa, H.; Hwang, C. C.; Yeom, H. W. Controlled Electron Doping into Metallic Atomic Wires: Si(111) $4 \times 1$-In. Phys. Rev. B: Condens. Matter Mater. Phys. 2010, 81, 075401.

(16) Lee, G.; Yu, S.-Y.; Shim, H.; Lee, W.; Koo, J.-Y. Roles of Defects Induced by Hydrogen and Oxygen on the Structural Phase Transition of Si(111)4 × 1-In. Phys. Rev. B: Condens. Matter Mater. Phys. 2009, 80, 075411.

(17) Shibasaki, T.; Nagamura, N.; Hirahara, T.; Okino, H.; Yamazaki, S.; Lee, W.; Shim, H.; Hobara, R.; Matsuda, I.; Lee, G.; et al. Phase Transition Temperatures Determined by Different Experimental Methods: Si(111) $4 \times 1$-In Surface with Defects. Phys. Rev. B: Condens. Matter Mater. Phys. 2010, 81, 035314.

(18) Uhm, S. H.; Yeom, H. W. Metal-Insulator Transition on the Si(111) $4 \times 1$-In Surface with Oxygen Impurity. Phys. Rev. B: Condens. Matter Mater. Phys. 2013, 88, 165419.

(19) Schmidt, W. G.; Babilon, M.; Thierfelder, C.; Sanna, S.; Wippermann, S. Influenceof $\mathrm{Na}$ Adsorption on the Quantum Conductance and Metal-Insulator Transition of the In-Si(111) $(4 \times$ 1) $-(8 \times 2)$ Nanowire Array. Phys. Rev. B: Condens. Matter Mater. Phys. 2011, 84, 115416.

(20) Park, S. J.; Yeom, H. W.; Min, S. H.; Park, D. H.; Lyo, I. W. Direct Evidence of the Charge Ordered Phase Transition of Indium Nanowires on Si(111). Phys. Rev. Lett. 2004, 93, 106402.

(21) Oh, D. M.; Wippermann, S.; Schmidt, W. G.; Yeom, H. W. Oxygen Adsorbates on the $\mathrm{Si}(111) 4 \times 1$-In Metallic Atomic Wire: Scanning Tunneling Microscopy and Density Functional Theory Calculations. Phys. Rev. B: Condens. Matter Mater. Phys. 2014, 90, 155432.

(22) Shim, H.; Lim, H.; Kim, Y.; Kim, S.; Lee, G.; Kim, H.-K.; Kim, C.; Kim, H. Initial Stages of Oxygen Adsorption on $\mathrm{In} / \mathrm{Si}(111)-(4 \times$ 1). Phys. Rev. B: Condens. Matter Mater. Phys. 2014, 90, 035420.

(23) Kim, T.-H.; Yeom, H. W. Topological Solitons versus Nonsolitonic Phase Defects in a Quasi-One-Dimensional ChargeDensity Wave. Phys. Rev. Lett. 2012, 109, 246802.

(24) Arguello, C. J.; Chockalingam, S. P.; Rosenthal, E. P.; Zhao, L.; Gutiérrez, C.; Kang, J. H.; Chung, W. C.; Fernandes, R. M.; Jia, S.; Millis, A. J.; et al. Visualizing the Charge Density Wave Transition in $2 \mathrm{H}-\mathrm{NbSe}_{2}$ in Real Space. Phys. Rev. B: Condens. Matter Mater. Phys. 2014, 89, 235115.

(25) Weitering, H. H.; Carpinelli, J. M.; Melechko, A. V.; Zhang, J.; Bartkowiak, M.; Plummer, E. W. Defect-Mediated Condensation of a Charge Density Wave. Science 1999, 285, 2107-2110.

(26) Farías, D.; Kaminski, W.; Lobo, J.; Ortega, J.; Hulpke, E.; Pérez, R.; Flores, F.; Michel, E. G. Phonon Softening, Chaotic Motion, and Order-Disorder Transition in Sn/Ge(111). Phys. Rev. Lett. 2003, 91, 016103.

(27) Anderson, P. W. Knight Shift in Superconductors. Phys. Rev. Lett. 1959, 3, 325-326.

(28) Abrikosov, A.; Gorkov, L. Contribution to the Theory of Superconducting Alloys with Paramagnetic Impurities. Sov. Phys. JETP 1961, 12, 1243-1253.

(29) Robertson, J. A.; Kivelson, S. A.; Fradkin, E.; Fang, A. C.; Kapitulnik, A. Distinguishing Patterns of Charge Order: Stripes or Checkerboards. Phys. Rev. B: Condens. Matter Mater. Phys. 2006, 74, 134507.

(30) Soumyanarayanan, A.; Yee, M. M.; He, Y.; van Wezel, J.; Rahn, D. J.; Rossnagel, K.; Hudson, E. W.; Norman, M. R.; Hoffman, J. E.
Quantum Phase Transition from Triangular to Stripe Charge Order in $\mathrm{NbSe}_{2}$. Proc. Natl. Acad. Sci. U. S. A. 2013, 110, 1623-7.

(31) Levy, N.; Burke, S. A.; Meaker, K. L.; Panlasigui, M.; Zettl, A.; Guinea, F.; Castro Neto, A. H.; Crommie, M. F. Strain-Induced Pseudo-Magnetic Fields Greater Than $300 \mathrm{~T}$ in Graphene Nanobubbles. Science 2010, 329, 544-547.

(32) Liu, Y.; Li, Y. Y.; Rajput, S.; Gilks, D.; Lari, L.; Galindo, P. L.; Weinert, M.; Lazarov, V. K.; Li, L. Tuning Dirac States by Strain in the Topological Insulator $\mathrm{Bi}_{2} \mathrm{Se}_{3}$. Nat. Phys. 2014, 10, 294-299. 